\title{
A BIOMECHANICAL MODEL OF THE LUMBOSACRAL JOINT DURING LIFTING ACTIVITIES
}

\author{
Charles K. Anderson,* Don B. Chaffin, $†$ Gary D. Herrin ${ }^{\dagger}$ and \\ LARRY S. MatTIIEWS $\ddagger$ \\ * Back Systems, Inc., 5495 Beltline Road, Suite 390, Dallas, TX 75240, U.S.A.; +Center for Ergonomics. \\ Department of Industrial and Operations Engineering, The University of Michigan, Ann Arbor. \\ MI 48109, U.S.A.; $¥ C 4002$ Outpatient Building, Box 054. University Hospital, The University of \\ Michigan, Ann Arbor, MI 48109, U.S.A.
}

\begin{abstract}
A biomechanical model of the lumbosacral region was constructed for the purpose of systematically studying the combined stresses and strains on the local ligaments, muscles and disc tissue during sagittal plane two-handed lifting.

The model was validated in two ways. The first validation was a comparison of experimental study results with model predictions. In general predictions compared very reasonably with observed values of several authors with the exception of strain predictions on the articular ligaments. Second, a sensitivity analysis was performed over a wide range of lifting tasks. The predicted stress/strain values followed anticipated patterns and were of reasonable magnitudes.

On the basis of the results of the sensitivity analysis it was concluded that typical lifting tasks can lead to excessive disc compressive forces, muscle moment generation requirements, and possibly lumbodorsal fascia strains. Conversely, annulus rupture of a healthy disc due to overstrain appears very unlikely.
\end{abstract}

\section{INTRODUCTION}

The prevalence and cost of industrially-related low back pain underscores the need for a better understanding of the manner in which such pain incidents arise. Of particular interest is the role played by variables such as type of task (e.g. lift, lower, etc.). magnitude of the load in the hands and posture of the worker while executing various tasks. It is generally recognized that a quantitative biomechanical model that predicts low-back tissue stress/strain on the basis of a set of task descriptors would be useful in this regard.

\section{MODEL DESIGN}

The purpose of this paper is to describe the development and validation of a biomechanical model of lifting for industry. The model was restricted to lifting tasks since these account for $40 \%$ to $60 \%$ of industrial low-back pain cases (Ayoub et al., 1978; Glover, 1960; Seager, 1959; Troup, 1965). Currently the model considers only the case of static sagittal-plane lifts. This case reasonably approximates the conditions of a slow, controlled lift in front of the body, which is often the recommended technique for lifting heavy objects in industry.

The model is designed to receive as input the task variables of discrete lifting postures and load in the hands. Predictions are then made of strain in the ligaments posterior to the lumbo-sacral joint centerof-rotation, the moment-generation requirement of the trunk erector musculature, compression on the

Received 29 May 1984; in revised form 14 January 1985. sacral endplate and strain in the posterior aspect of the outermost layer of the annulus. These tissue load parameters were selected on the basis of their purported role in the genesis of low-back pain. The ligaments that were included in the model were the following;

(1) lumbodorsal fascia,

(2) interspinous/supraspinous ligament,

(3) articular ligaments,

(4) ligamentum flavum, and

(5) iliolumbar/sacrolumbar ligaments.

The multifidi and erector spinae were considered as the primary trunk erector muscles at the $\mathrm{L} 5 / \mathrm{S} 1$ level. Figure 1 illustrates the geometric representation of the low-back ligament elements. The ligaments and muscles are represented by lines of action attached to the two vertebra at specific points in a manner adapted from the models of Schultz and his colleagues (e.g. Takashima et al., 1979).

\section{MODEL DEVELOPMENT}

A number of biomechanical models exist which allow one to predict one or another of the tissue load indices though no one model concurrently predicts all of the indices mentioned above. For instance there are models which;

(1) estimate moments and forces about the L5/S1 joint given a load in the hands and a particular body posture (e.g. Garg and Chaffin, 1975; Schultz et al., 1982);

(2) estimate strain in the ligaments given the relative orientation of L5 and the sacrum (e.g. Seireg and Arivikar, 1975; Takashima, et al, 1979; Yettram and Jackman, 1980), and

(3) estimate the strain on the outermost layer of the 


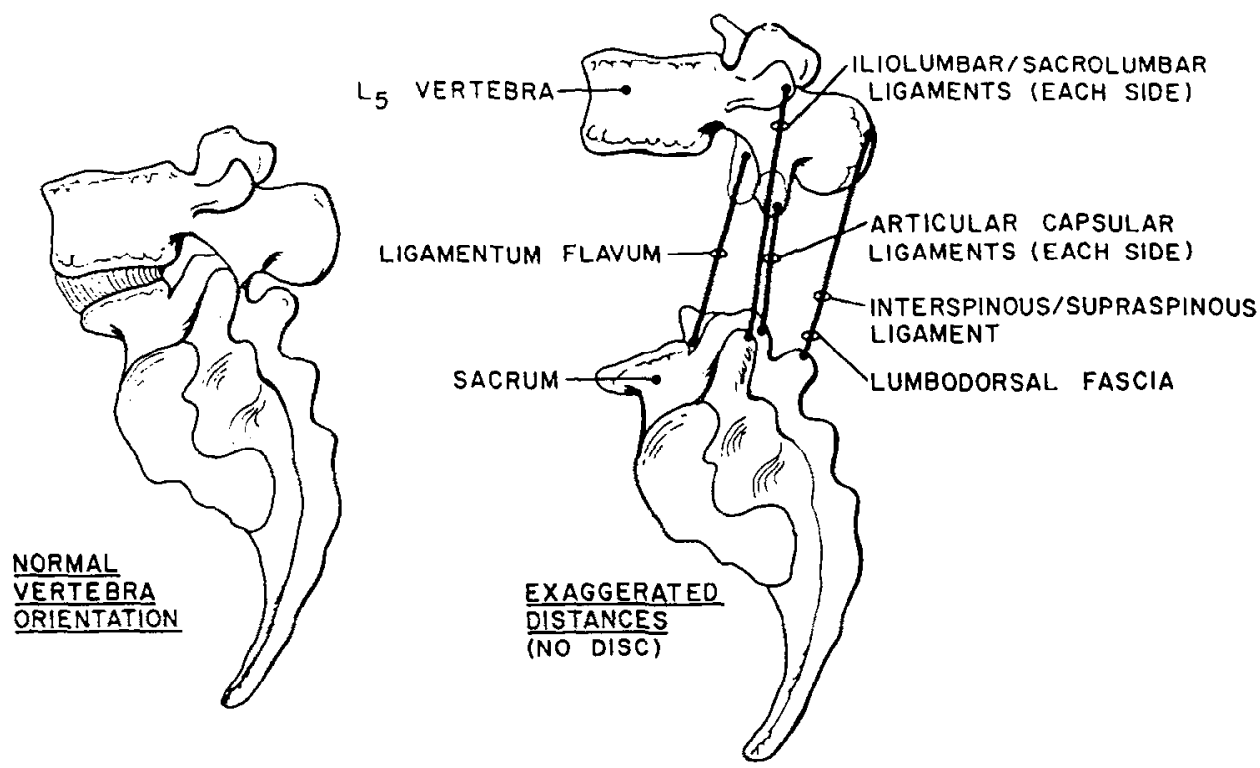

Fig. 1. Geometric representation of the low-back ligament elements.

annulus for a given disc compression (e.g. Broberg and von Essen, 1980; Broberg, 1983; Hickey and Hukins, 1980; Spilker, 1980).

To be specific, the whole-body models consider only disc compression but ignore the strain on the ligaments and annulus. The ligament models, on the other hand, presume a vertebral orientation of isolated motion segments and predict strains. These models do consider the disc but treat it in a simplified manner that does not allow concurrent estimation of strain in the layers of the annulus. The disc models predict strain in the layers of the annulus but are divorced from the rest of the body and are designed for simple loading cases, such as pure compression or bending moments.

After review of the models which were available it became evident that three distinct efforts would be necessary to construct a more integrative prediction model. These three efforts would be to develop;

(1) a kinematic model which predicts LS and S1 orientation given whole-body posture and load in the hands;

(2) an L5/S1 biomechanical model which incorporates the contribution to restorative moment due to passive tissue strain, and

(3) a disc model incorporated within the LS/S1 motion-segment model which estimates annular strain under conditions of combined compression, flexion and shear (as opposed to isolated compression).

What follows is a discussion of the methodology employed in the model. The reader interested in further details should consult Anderson (1983).

\section{Vertebral reorientation kinematic model}

Data regarding the relative positions and angular orientations of the L5 and SI vertebra are necessary for the calculation of moments and forces acting on the lumbosacral tissues. Because of the difficulty of directly determining the angular orientation of these two vertebra during normal lifting activities it was necessary to develop prediction models that were functions of readily-observable variables. Review of the literature suggested that torso angle, knee angle and load in the hands would be the most important parameters to include. It was also found that the data needed to construct such models did not currently exist.

For this reason, extensive data was collected (Anderson, 1983) on four subjects from which predictive equations of sacral rotation and $\mathrm{LS}$ rotation relative to the sacrum were derived as functions of torso angle, knee angle, and load in the hands while lifting.

Statistical analysis revealed that load in the hands gave a negligible improvement in the predictive efficiency of the equations, therefore it was eliminated from the final set of independent variables. The form of the final regression equations are presented graphically in Figs 2 and 3. L5/S1 relative rotation was scaled to the percent of the maximum rotation so that individuals of varying flexibility could be better accomodated. The equations are as follows

$$
\begin{aligned}
S= & -17.519-0.11863 T+0.22687 K+0.11904 e \\
- & 2 T^{*} K+0.499 e-2 T^{2}-0.753 e-3 K^{2} \\
L S= & 32.642+1.3096 T-0.74916 e-1 K \\
& +0.00069 T^{*} K-0.00652 T^{2}-0.0007 K^{2}
\end{aligned}
$$

where $S=$ sacral rotation from position when standing erect (in degrees); $L S=$ percent of maximum $\mathrm{L} 5 / \mathrm{S} 1$ relative rotation; $T=$ torso rotation from erect standing (in degrees); $K=$ included knee angle (in degrees).

It can be seen that $\mathrm{S} 1$ rotation (Fig. 2) and L5 


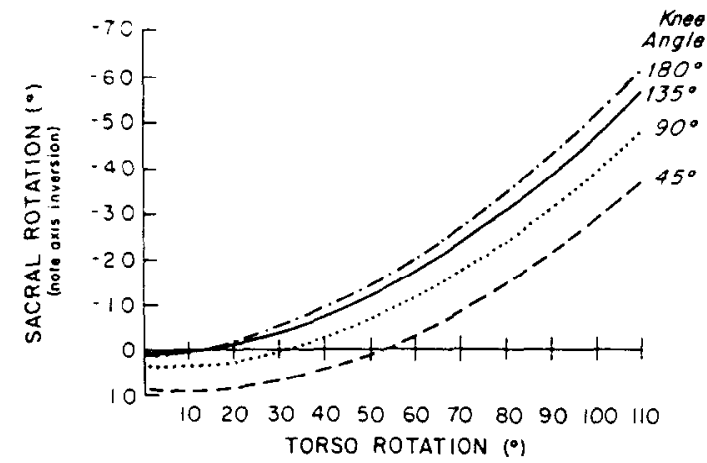

Fig. 2. Predicted sacral rotation vs torso rotation.

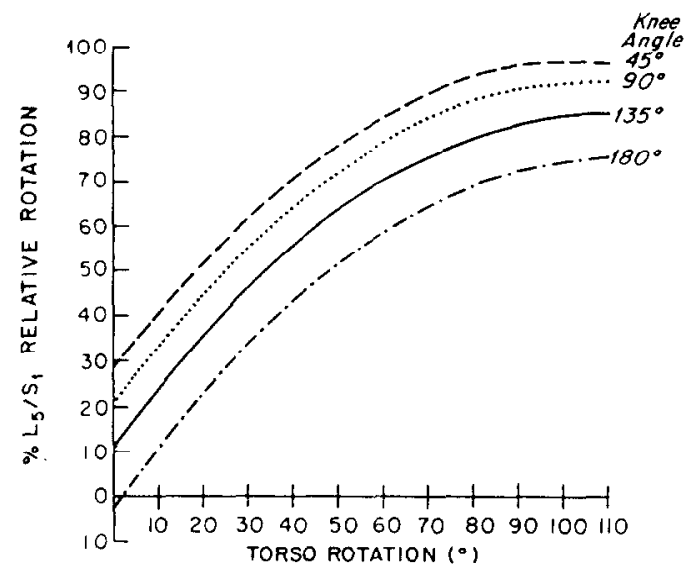

Fig. 3. Predicted percent of maximum L5/SI relative rotation vs torso rotation.

rotation relative to $\mathrm{S} 1$ (Fig. 3) both increase with torso angle but the shape of the curves are distinctly different. The curves show that the first $30^{\circ}$ of trunk flexion is primarily accomplished though rotation in the lumbar spine but shifts to predominately hip rotation as torso rotation increases beyond approximately $30^{\circ}$. Decreasing knee angle (i.e. bending the knees) shifts the curve for S1 rotation (Fig. 2) down and $\mathrm{L} 5 / \mathrm{S} 1$ relative rotation up (Fig. 3). Performance statistics are presented in Table 1. This portion of the project is discussed at length in Anderson et al. (in press).

Table 1. Summary of coefficients of determination $\left(r^{2}\right)$ and standard errors for predictive equations of sacral rotation and percent of maximum L5/Si relative rotation

\begin{tabular}{llc}
\hline \multicolumn{1}{c}{ Equation } & $r^{2}$ & $\begin{array}{c}\text { Standard } \\
\text { error }\end{array}$ \\
\hline $\begin{array}{l}\text { Sacral rotation } \\
\text { Percent of maximum } \\
\begin{array}{l}\text { LS/Si relative } \\
\text { rotation }\end{array}\end{array}$ & 0.89 & $7.43^{\circ}$ \\
\hline
\end{tabular}

\section{Whole-body model enhancement}

Estimation of stresses and strains on the lumbosacral tissues for a specified lifting task was accomplished by considering the balance of forces and moments required by static equilibrium conditions about the L5/S1 joint. The moment equilibrium condition requires that the resultant and restorative moments about the joint be balanced. The resultant moments in the case of lifting act to flex the upper body forward around the $\mathrm{L} 5 ; \mathrm{S} 1$ joint while the restorative moments act to resist the forward flexion. The resultant moment has two components:

(1) moment due to the load in the hands. and

(2) moment due to the weight of the body segments above L5/SI.

The restorative moment is modelled as having four components:

(1) moment due to abdominal pressure,

(2) moment due to resistance of the disc to bending,

(3) moment due to resistance of the ligaments to strain,

(4) moment due to muscle contraction and passive resistance of muscle.

Mathematically the moment equilibrium condition is expressed as follows

$$
M_{W}+M_{B}=M_{A}+M_{L}+M_{M}+M_{D}
$$

where $M_{W}=$ moment due to load in the hands; $M_{B}=$ moment due to body weight: $M_{A}=$ moment due to abdominal pressure; $M_{L}=$ moment due to ligament strain; $M_{M}=$ moment due to muscle and $M_{0}=$ moment due to disc resistance to bending.

The elements on the right side of equation (3) constitute the resultant load moment in flexion while the elements on the left side represent the restorative extensor moment about the $\mathrm{L} 5 / \mathrm{S} 1$ center of rotation. It will be seen in the discussion below that all parameters of the moment equilibrium equation except the moment due to muscle can be computed from information in the literature.

The force equilibrium conditions for compression and shear can be expressed as follow's

$$
F_{S E}=F_{H}+F_{B}+F_{A}+F_{L}+F_{M}
$$

where $F_{\text {SE }}=$ force acting on the sacral endplate; $F_{W}$. $=$ force due to load in the hands; $F_{B}=$ force due to body weight above $\mathrm{L} 5 / \mathrm{S} 1 ; F_{A}=$ force due to abdominal pressure (compression only); $F_{L}=$ force due to ligament strain and $F_{M}=$ force due to muscle contraction.

The equation has the same form whether the force is compressive or shear in nature. Force due to load in the hands is given. The remaining parameters except for force on the sacral endplate can be computed from data in other sources.

Hence there are three equations and three unknowns. The three unknowns are;

(1) moment due to muscles,

(2) compressive force at the sacral endplatic and 
(3) shear force at the sacral endplate.

The general algorithm for solving the equilibrium equations for the three unknowns is depicted in Fig. 4. The process will be briefly described here and then explained in more detail below. In Fig. 4 it can be seen that the first step is to use photographic information about the body posture to locate the body links in space. Moments due to load and body weight about all the joint centers in the body are calculated during this step. Postural information and moment estimates from the whole-body model are transferred to the lumbosacral ligament/muscle model for further analysis at this point. This is accomplished by the following three steps:

(1) Torso angle and knee angle are used to predict the orientation of L5 and S1 which is required for calculating moments about the L5/S1 joint.

(2) The intra-abdominal pressure restorative moment about the $\mathrm{L} 5 / \mathrm{S} 1$ joint is predicted from data of others.

(3) The moments due to ligament resistance and muscle contraction are calculated. Results of these calculations are then transferred to the disc model in order to estimate strain on the posterior annulus.

The description of the steps involved in the lumbosacral ligament/muscle model algorithm are expanded below.

\section{Resultant moments}

In order to calculate all of the moments it is necessary to know the spatial location of the $\mathrm{L} 5 / \mathrm{S}$ l center-of-rotation. Body posture information obtained from photographs in conjunction with estimates of link lengths are used to first derive the spatial location of the sacrum. Link lengths are scaled as percents of stature or are measured from palpable body landmarks. The sacral rotation and $\mathrm{L} 5$ rotation relative to the sacrum are then predicted by entering knee and hip (torso) angles into the kinematic model equations (equations 1 and 2). The joint-center is then located under the assumption that it is at the center of the disc separating the two vertebrae.

Load in the hands and body weight. Calculation of moments due to load in the hands and body weight are relatively straightforward once the $\mathrm{L} 5 / \mathrm{S} 1$ joint center is located. The algorithm incorporated in the wholebody biomechanical model described by Garg and Chaffin (1975) was used for the calculations. Essentially moment arms are calculated for the load in the hands and the body segments above L5/S1 and then multiplied by the respective weight of the segment. The load in the hands and body weight above L5/S1 are also decomposed into normal and shear forces acting on the sacral endplate using the sacral endplate angle predicted from equation (1).

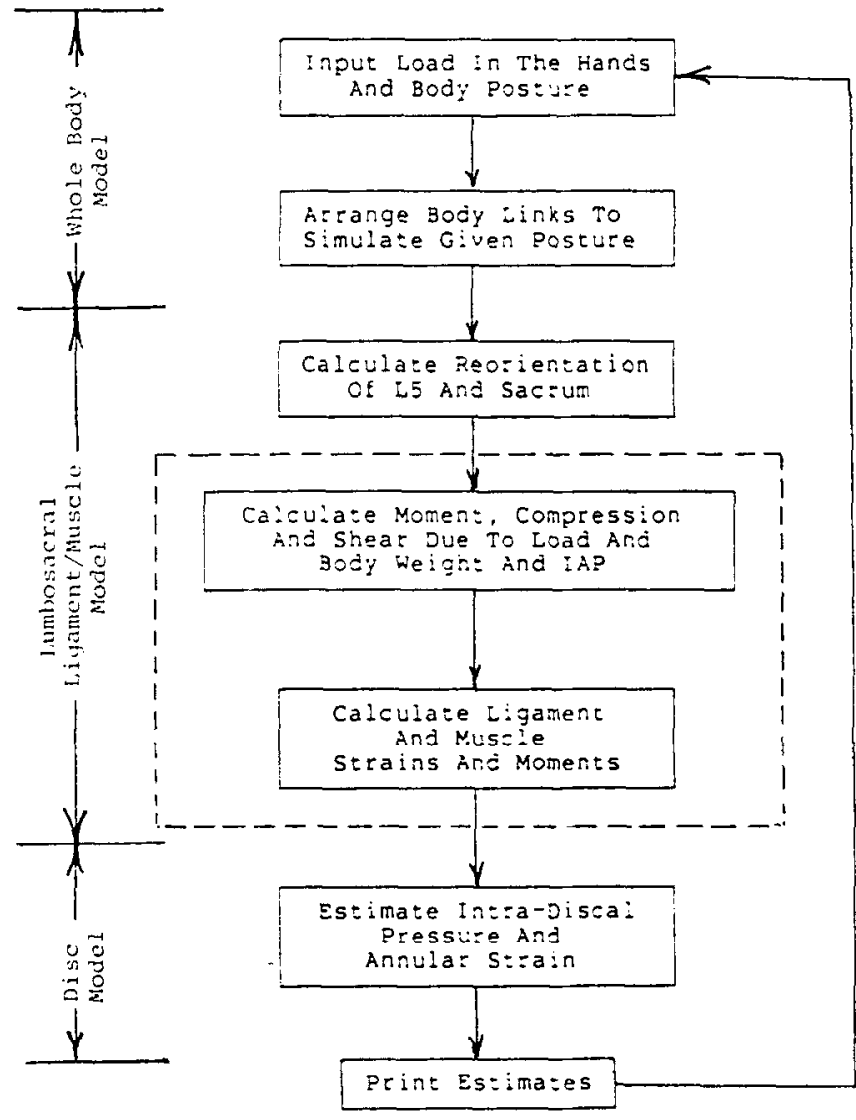

Fig. 4. Flow diagram of lumbosacral joint biomechanical model algorithm. 


\section{Restoratice moments}

The static equilibrium assumption dictates that the total restorative moment must equal the resultant moment. but the question arises as to how the moment is partitioned among the four elements, namely intraabdominal pressure, disc resistance, ligament resistance and muscle contraction. Intra-abdominal pressure and disc resistance calculations are not contingent on ligament strain or muscle activity, so their contribution is considered first.

Abdominal pressure. The moment contribution due to abdominal pressure was calculated in the same manner as described in Chatfin (1969) except that an equation was developed that was a function of $L 5 / S 1$ resultant moment rather than the external load moment about the hip. The equation was derived, as was the original, from data presented by Morris et al. (1961). It explained $49^{\circ}$ of the variance in the data of Morris et al. Essentially, abdominal pressure is considered a function of torso angle and resultant moment about the L5/St joint. The equation derived was

$$
I A P=e^{-3.0145}(\cos T)^{-0.25166} M^{0.9359}
$$

where $l A P=$ intra-abdominal pressure $(\mathrm{mmHg}$ ); $T=$ torso angle (rad) and $M=$ resultant external moment about $\mathrm{L} 5 / \mathrm{S} 1(\mathrm{Ncm})$ due to load in the hands and body weight above the LS/S1 disc. The torso angle is taken as the included angle formed by the shoulder, hip and horizontal through the hip.

The pressure is assumed to act perpendicular to the endplate as a tension element across the disc at a distance of 7-15 cm, as estimated from an equation developed by Fisher (1967) and described in detail in Chaffin and Andersson (1984). The pressure and distance are then multiplied to obtain the moment about L5/S1 due to abdominal pressure.

Disc resistance to bending. The moment contribution due to disc resistance was calculated through utilization of a regression equation based on information from Schultz et al. (1979), Panjabi et al. (1977) and Rolander (1966). The equation of disc moment as a function of disc bending is

$$
M_{D}=e^{1.634} * e^{21.026 R}
$$

where $M_{D}=$ moment due to disc resistance to bending $(\mathrm{Ncm})$ and $R=$ L5 rotation relative to $\mathrm{Sl}$ (rad). The coefficient of determination was 0.981 using five data points. There was assumed to be negligible force on the endplate generated by the disc resistance to bending. Equation (2) is used to predict the relative rotation. The value of $R$ is obtained in rad by assuming that $100 \%$ relative rotation corresponds to $12^{\circ}$ or 0.21 rad.

Ligament strain. The moment due to strain in the ligaments was calculated by the following steps:

(1) estimate the elongation of each ligament connecting $L 5$ and the sacrum and convert it to a strain by dividing by the resting length (see Appendix $A$ for resting length values);

(2) calculate the force from force-strain curves derived from the literature (see Appendix B for equations) and

(3) convert the forces into moments based on the lines of action of the ligaments as determined by the locations of the attachmene points of the ligaments to the vertebra and the location of the joint center.

Attachment points for all ligaments and estimated force-strain curves for ligaments currently not studied were generously provided by Dr. A. Schultz (personal communication, 1981). Each ligament force was also decomposed into a normal and shear force on the sacral endplate.

Muscle contribution. Whatever amount of the resultant moment remained after subtracting the moment due to intra-abdominal pressure, disc resistance to bending and ligament resistance to strain was attributed to muscle contraction. The moment due to the musculature was assumed to be partitioned between the multifidi and erector spinae in proportion to ratio of their cross-sectional areas (taken as 13.2:18.6 ratio). The muscle forces were multiplied by their moment arms to obtain the moment due to muscle contraction and then decomposed to obtain normal and shear forces on the sacral endplate based on insertion angles.

The reader will note that calculation of ligament strain requires knowledge of the L5/SI disc height, which in turn is a function of the compressive force acting on the joint. Increased compressive force, for instance, causes the disc to decrease in height, which concomitantly decreases the distances between the ligament attachment points. This in turn decreases the strain in the ligaments and thereby the ligament compressive contribution on the disc. When the ligament contribution to the moment changes, the remainder of the resultant moment which is assumed to be compensated by muscle contraction changes in the opposite direction. This change also affects the muscle contribution to compression and shear on the disc. Hence an iterative approach is required to simultaneously estimate the disc height and in turn the stress/strain parameters about the L5/SI motion segment. The basic outline for the iteration algorithm is shown in Fig. 5. This figure gives more detail for the portion of the algorithm enclosed in the dashed box shown in Fig. 4.

A first estimate of compressive force on the disc is obtained by assuming no ligament contribution and contribution from the muscles occurring through a single muscle equivalent which operates perpendicular to the endplate at a distance of $5 \mathrm{~cm}$, as described in Chaffin (1969) and Chaffin and Andersson (1984). The compressive force due to muscle contraction is added to compressive force due to load in the hands, body weight and intra-abdominal pressure. The sum of the compressive force components is entered into an equation of disc height vs compressive force to be described in the next sub-section. Ligament strain can then be calculated under these initial conditions and a muscle moment can be determined. A refined estimate 


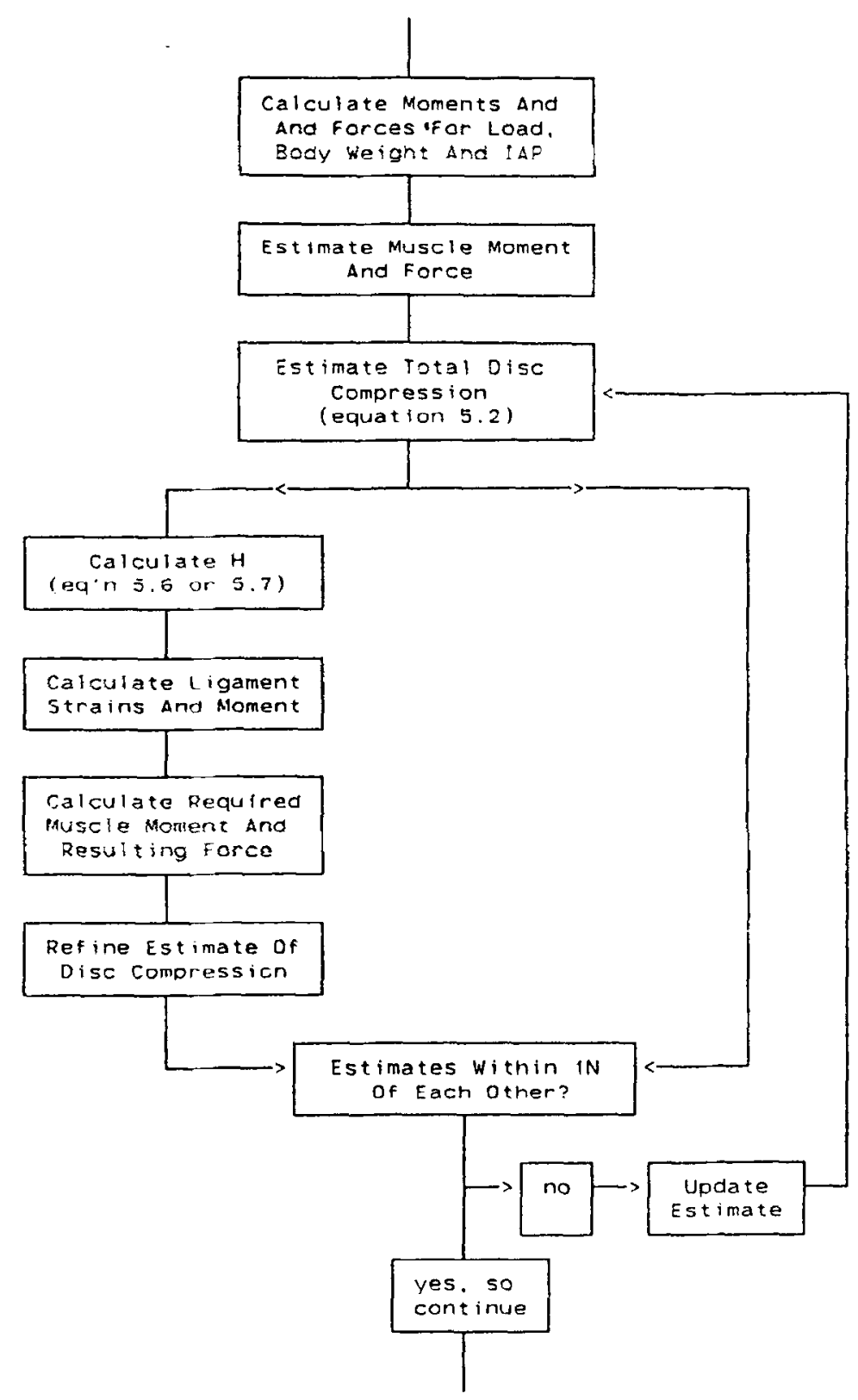

Fig. 5. Iteration loop for determination of disc compressive force.

of compressive and shear forces is thus obtained. If the revised compressive force does not match the compressive force associated with the current disc height, the estimate is adjusted in the appropriate direction and another iteration is performed. The cycle is continued until the two compressive force estimates are within $1 \mathrm{~N}$ of each other. The final compressive and shear forces along with the amount of flexion in the disc are then passed to the disc model.

\section{Disc model extension}

The disc model of Broberg and von Essen (1980) was extended (Anderson, 1983) so that the complex loadings of compression, flexion and shear derived from the lumbosacral ligament/muscle model could be analyzed in terms of strain on the annulus, which may be an indicator of the risk of disc rupture. Parameter values used in Broberg and von Essen's disc model are presented in Appendix C.

The effects of combined compression, shear and bending on annular strain were assessed by estimating the contribution to intra-discal pressure due to each component of the load and then summing the contributions. Equations are given in Anderson (1983) along with sources of data. The 'effective' intra-discal pressure was then entered into an equation derived from the compression model adapted from Broberg and von Essen which describes the relationship between model 
predictions of strain in the outermost layer of the annulus (assuming parallel endplates) and the intradiscal pressure. A second estimate of the strain is derived by calculating the distance between the attachment points on the re-oriented endplates for the fiber whose midpoint is most-posterior. The actual strain on the fiber is taken as the maximum of these two estimates. If strain is actually determined by reorientation due to shear and bending, the straight-line distance will be greater, otherwise the strain due to bulge will be the major factor and the first estimate will be larger.

The additive model is supported by the results of Nachemson (1963) who found the rise in intra-discal pressure due to bending was independent of the compressive load. This was the only study available which quantified the effects of combined loads on intra-discal pressure, so the existence of any synergistic effect due to shear was unknowr.

Upon analyzing the performance of Broberg and von Essens' model with fiftieth-percentile disc measurements under pure compression, it was noticed that relaxation of the model constraint of a rigid endplate assumed by Broberg and von Essen (1980) to one of a flexible endplate yielded a disc model that better replicated observed disc behavior seen in experimental conditions. The endplates were allowed to bulge spherically in such a way so as to keep a constant volume in the disc for a given annular bulge. The magnitude of the annular bulge was predicted from an equation fit to the data of Brown et al. (1957) which predicted annular bulge from a decrease in disc height. In a later article, Broberg (1983) described a morecomplex disc model in which he also allowed the endplates to bulge, but the degree of bulging is mathematically determined by model constraints. In both cases the extended models performed much better than the original model in terms of congruence with published data, thus justifying the added geometric complexity.

\section{MODEL VALIDATION}

Two forms of validation were performed on the integrated model. The first form of validation involved comparing predicted and observed stress/strain values. Cadaver and in vivo experimental studies giving observed values of low-back related stresses and strains were gleaned from the literature and simulated with the integrated model or components thereof as appropriate to the study. The predicted values obtained from the simulations were then compared to the observed values from the respective study.

The second form of validation involved study of ligament and posterior annulus strains, erector musculature moment requirements and disc compressive loads for a range of postures and loads in the hands.

\section{Validation with data from the literature}

Three basic sets of comparisons were performed. The first set was related to the mechanical behavior of an isolated functional spinal unit (FSU) as it is flexed. The primary parameter observed was the moment created by the ligaments and disc as they resist flexion. Excellent agreement was obtained for the comparison of predicted values to the observed values of composite ligament moment under flexion presented by Panjabi et al. (1977) and Schultz et al. (1979). The maximum deviation between predicted and observed values was $200 \mathrm{Ncm}$ over a range of $0-2600 \mathrm{Ncm}$. Less favorable results were obtained when simulating the study reported by Adams et al. (1980). Adams et al. observed a ligament moment of $4983 \mathrm{Ncm}$ at full-flexion while predicted ligament moment at full flexion was approximately $2100 \mathrm{Ncm}$.

The second set of comparisons concerned strain levels of the ligaments under conditions of maximal LS/S1 forward flexion in relation to their elastic limits (see Table 2). In general maximal predicted ligament strains as compared to experimentally derived elastic limits suggested that the lumbodorsal fascia was the only passive tissue at any degree of risk. It was also noted that the articular ligament had an unreasonably high predicted strain value. The lumbodorsal fascia strain response agrees with Farfan and his colleagues who have suggested that the lumbodorsal fascia plays a major role in resisting forward flexion (Bazergui, et al, 1978; Gracovetsky et al. 1981). The result does not correspond to the results of Rissanen (1960) and Adams et al. (1980) who suggest that ruptures of the supraspinous/interpinous ligament are fairly common.

The simulation results for the articular ligaments indicated that the tissue had to sustain maximal strains of $100-130 \%$ strain (see Table 2), which is unrealistic. More to the point, these results suggest that the manner in which these ligaments are modelled is not accurate. The information needed to improve the model consists of better approximations of the attachments to the vertebra, and more realistic values for resting length. The shortcoming of the current model in this regard does not affect the other parameter predictions, though, due the very small contribution to

Table 2. Comparison of predicted percent of strain at $100^{\circ}$. flexion and observed elastic limits by ligament

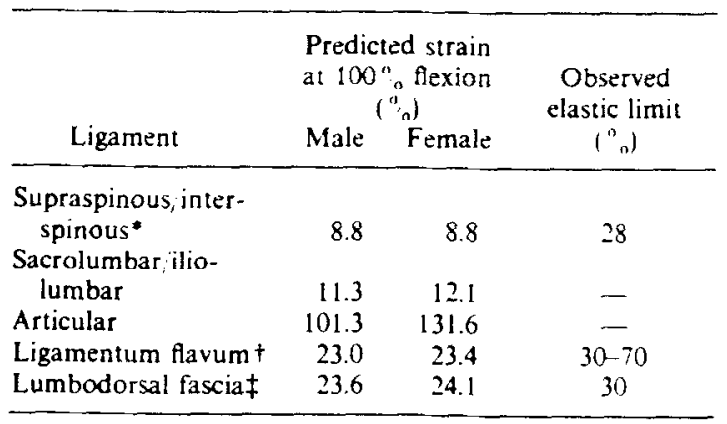

* Waters and Morris (1973).

+ Nachemson and Evans (1968).

† Bazergui et al. (1978). 
the restorative moment offered by the articular ligaments under current assumptions.

The third set of comparisons involved intra-discal and intra-abdominal pressure estimates under various load and posture conditions. For this, studies by Andersson et al. $(1976,1977)$ were simulated. Observed and predicted values showed very good agreement for intra-abdominal pressure (Fig. 6), but were not in as close accord for intra-discal pressure (Fig. 7). Predicted values of intra-discal pressure were higher than those observed in the two studies. This may be due to the fact that potential load-bearing capability of the facet joints is not incorporated in the model. It is important to also note that very little quantitative information about body posture other than vertical hand location was given in the second of the two articles so the simulation may not be totally representative of the actual posture used by the subjects.

The results of these three sets of comparisons were used iteratively to adjust the model parameters within the bounds of physiologically-reasonable values so as to obtain the best overall correspondence to behavior seen in experimental studies. In general, the comparative validations suggested that the model yields predicted values that are reasonably close with the exception of the articular ligaments and possibly the intra-discal pressure. In order to better model the articular ligaments it will be necessary to improve the information describing the ligament resting length, attachment points and force-deformation curves. In the meantime the other parameters that are predicted

\section{INTRA-ABDOMINAL PRESSURE VS. TRUNK ROTATION} COMPARISON OF OBSERVED AND PREDICTED

\section{VALUES FOR A $200 \mathrm{~N}$ LOAD}

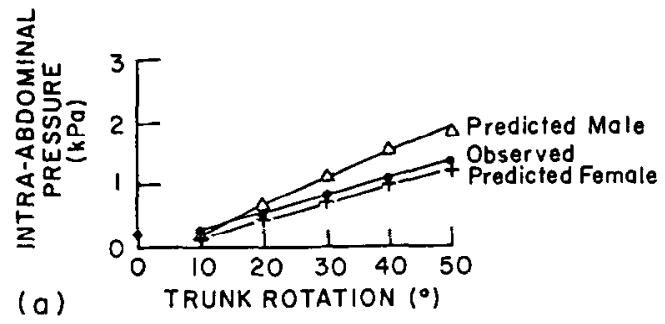

COMPARISON OF OBSERVED AND PREDICTED VALUES FOR INTRA-ABDOMINAL PRESSURE VS. LOAO IN THE HANDS AT $30^{\circ}$ TRUNK ROTATION

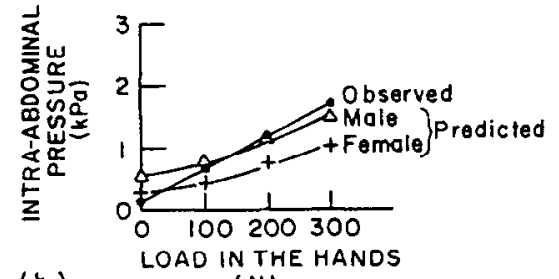

(b) yield reasonable values based on comparisons to experimental studies.

\section{Analysis of posture and load}

Performance of the model was also studied by analyzing the effects on tissue stress/strain of inputting body postures and loads in the hands that correspond to the wide range of conditions found in industry. In specific, the sensitivity analysis consisted of model simulations of the following combinations of parameters:

(1) torso rotation: 0 (erect), 30, 60 and $90^{\circ}$,

(2) knee angle: 180 (straight-leg), 120 and $60^{\circ}$, and (3) load in the hands: $O N$ and $500 \mathrm{~N}$.

The primary predicted values of interest were ligament strains, strain on the posterior aspect of the annulus, contributions to the restorative moment due to the ligament structure, muscle structure, disc and abdominal pressure and contributions to the disc compression due to load in the hands, body weight above $\mathrm{L} 5 / \mathrm{S} 1$, ligaments, muscles and abdominal pressure. The results are presented below for each category of predicted values.

\section{Ligament strain results}

In general it was found for all of the ligaments that:

(1) strain monotonically increased with torso rotation,

(2) strain increased with decreasing knee angle, and

(3) there was more strain at $0 \mathrm{~N}$ than at $500 \mathrm{~N}$ (assuming the muscles are relaxed).

COMPARISON OF OBSERVED AND PREDICTED VALUES FOR INTRA-OISCAL PRESSURE vS. TRUNK ROTATION AT A $200 \mathrm{~N}$ LOAD

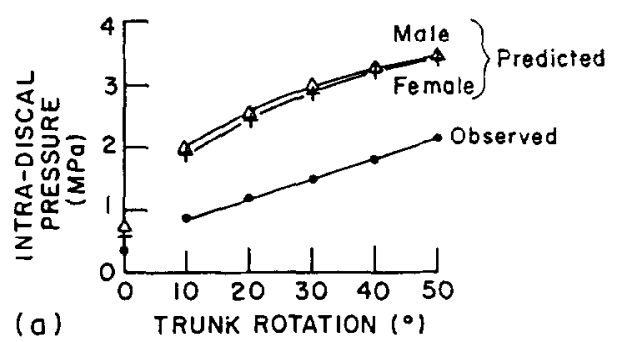

COMPARISON OF OQSERVED ANO PREDICTED VALUES FOR INTRA-DISCAL PRESSURE YS. LOAD IN THE HANDS AT $30^{\circ}$ TRUNK ROTATION

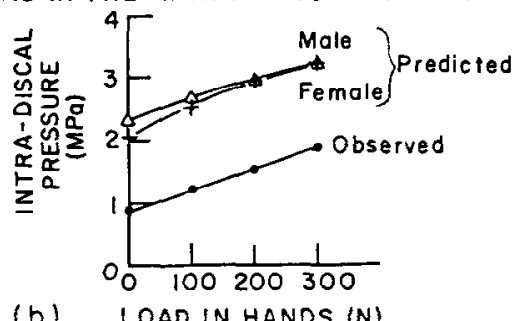

(b) LOAD IN HANOS (N)

Fig. 6. Comparison of observed (Andersson et al., 1976) and predicted intra-abdominal and intra-discal pressures. (a) $30^{\circ}$ trunk rotation. (b) $200 \mathrm{~N}$ load in the hands. 

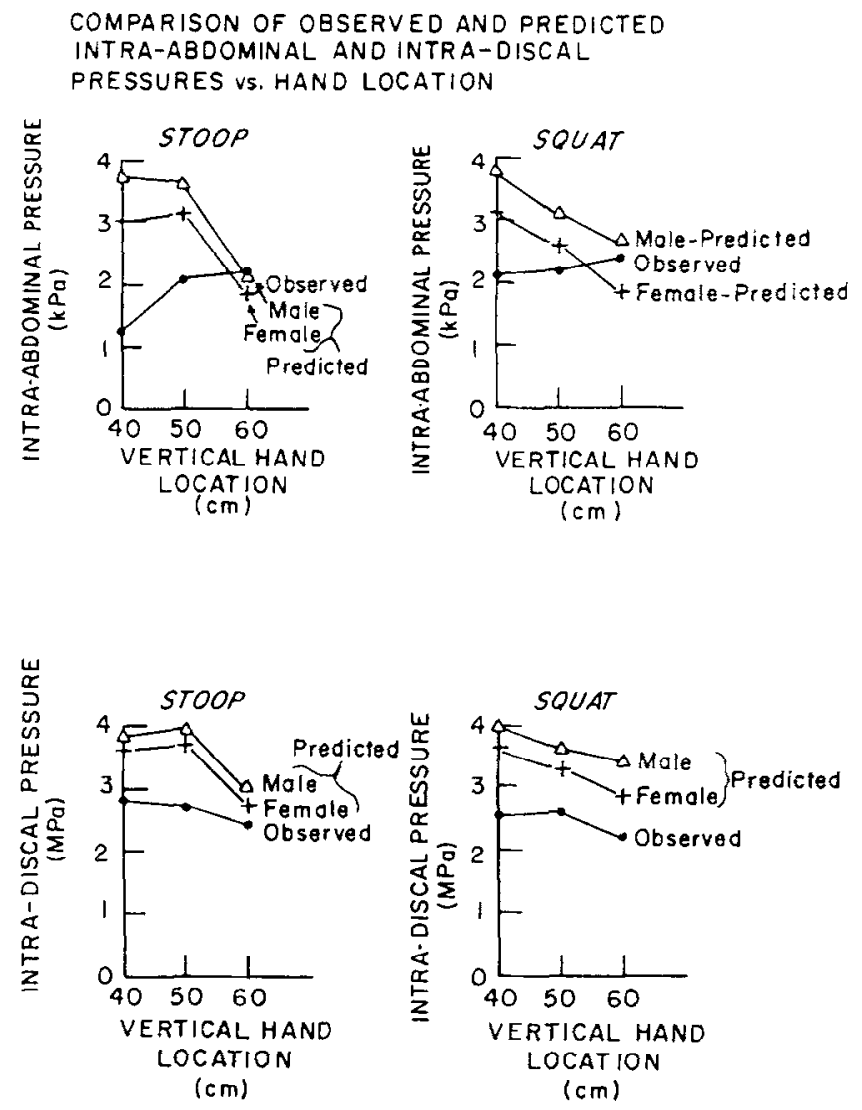

Fig. 7. Comparison of observed (Andersson et al, 1977) and predicted intra-abdominal and intra-discal pressures vs hand location.

It would be expected that ligament strains would increase with torso rotation because the L5 and S1 vertebrae separate with rotation. The separation oocurs more rapidly as the knees are flexed (see Figs 2 and 3) so it is not surprising that strain increases with decreasing knee angle. The reason there is more strain at $0 \mathrm{~N}$ than at $500 \mathrm{~N}$ is that the disc separating the vertebra is increasingly compressed with increasing load in the hands which in turn means that the vertebral attachment points of the ligaments are closer together. Therefore maximum strain for a given torso rotation angle occurs with no load in the hands assuming the muscles are relaxed.

\section{Posterior annulus strain results}

The following observations were made:

(1) strain increases with torso rotation but never goes above about $2.7 \%$ under any condition studied and

(2) strain increases significantly with increases in load in the hands $\left(1.5 \%\right.$ vs $2.7^{\circ}$ for $0 \mathrm{~N}$ and $500 \mathrm{~N}$ respectively).

These strain estimates fall significantly below strain values derived from maximum elongation data of annulus samples tested along the axis of the fibers (Galante, 1967). Galante's data suggested that the elastic limit for annular fibers is approximately $31.5^{\circ}$ o with a standard deviation of $1.8_{, 0}^{\circ}$. This implies that the annulus does not reach the elastic limit even under loads which could cause failure of the functional spinal unit. This agrees with the observation that the endplates tend to fracture first under maximal loading conditions (Brown et al., 1957; Perey, 1957; Rolander, 1966).

\section{Restoratice moment results}

The components of the restorative moment due to muscles, ligaments and abdominal pressure are predicted based on varied torso rotations and at $0 \mathrm{~N}$ and $500 \mathrm{~N}$ loads on the hands. The predictions for straight legs are graphed in Fig. 8. Knee angle has virtually no effect on the restorative moments, so only one knee angle $\left(180^{\circ}\right)$ is displayed. The moment due to disc bending was so small that it was not included on this graph. It can be seen that generally the moment duc to muscle contraction predominates under all conditions, especially with larger load. The moment due to abdominal pressure had the second largest effect, and the moment due to ligament resistance was the smallest. The magnitude of the moments generally increases for all components as the trunk is flexed, though for muscle contraction the moment stops increasing between 60 and $90^{\circ}$ under $0 \mathrm{~N}$ load and in some cases begins to fall. 

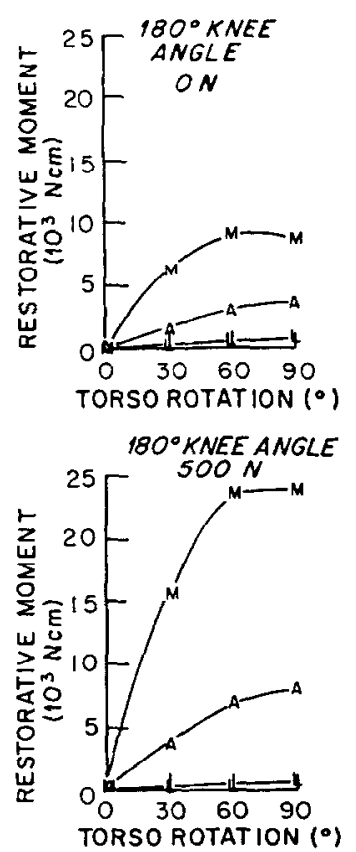

Fig. 8. Restorative moment vs torso rotation by element at $0 \mathrm{~N}$ and $500 \mathrm{~N}$ load in the hands with $180^{\circ}$ knee angle (straight leg).

The percent of restorative moment for each element vs torso rotation is plotted in Fig. 9. It can be seen that percent of the moment attributable to muscle contraction continually decreases after $30^{\circ}$ if not before, while the percent of the moment due to ligament tensions increases. The drop-off of the moment generated by
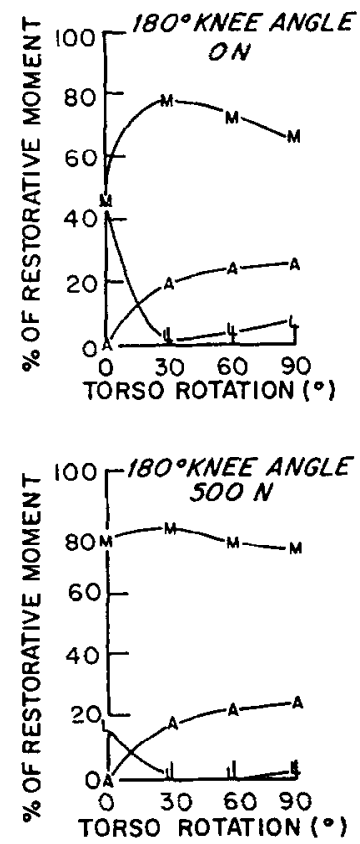

Fig. 9. Percent of restorative moment vs torso rotation by element at $0 \mathrm{~N}$ and $500 \mathrm{~N}$ load in the hands with $180^{\circ} \mathrm{knee}$ angle (straight leg). $\mathrm{L}=$ ligamental structure, $\mathrm{M}=$ muscle structure, $\mathrm{A}=$ abdominal pressure. muscle contraction is related to the non-linear response of the moment due to ligament tension as a function of torso rotation. Figure 8 shows how the ligament moment increases more-rapidly with increasing rotation. The percent of the moment due to abdominal pressure remains relatively constant.

It is interesting to note that under the no-load condition the ligaments play a much more important role in resisting the resultant moment than under a $500 \mathrm{~N}$ load. This follows from the observation that load in the hands decreases the disc height and thus reduces ligament strain.

\section{Muscle strength limit results}

Muscle moment requirements on the trunk extensors were also compared to the moment-producing capabilities (i.e. strength) of the working population. The procedure is described in Garg and Chaffin (1975). In short, the moment requirement is compared to the distribution of moment strengths for the population at the given joint angles. The percent of the population who have strengths below the requirement are taken to be the percent of the population who would be incapable of the trunk extensor moment requirement. For the conditions of this sensitivity analysis it was found that at the $500 \mathrm{~N}$ load up to $20 \%$ of the male population and up to $60 \%$ of the female population would be incapable of the trunk extensor moment requirement depending on the iorso angle (see Fig. 10).

\section{Disc compression results}

Disc compression was computed by calculating the contribution due to the load in the hands, body weight, muscles, ligaments and abdominal pressure and summing according to equation (4) to obtain the disc compression for the range of torso rotations, knee angles and loads in the hands. The component values are shown in Fig. 11 as a function torso rotation and load in the hands for the straight-leg condition. The reader will recall that the abdominal pressure acts as a tension across the disc and therefore appears in Fig. 11 as a negative compression.

The component values expressed in terms of percent of total compression for the given condition are shown in Fig. 12. As with restorative moment, knee angle has

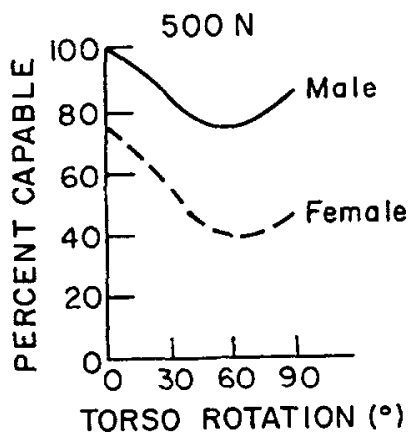

Fig. 10. Percent of the workforce capable of a $500 \mathrm{~N}$ lift vs torso rotation from erect posture. 


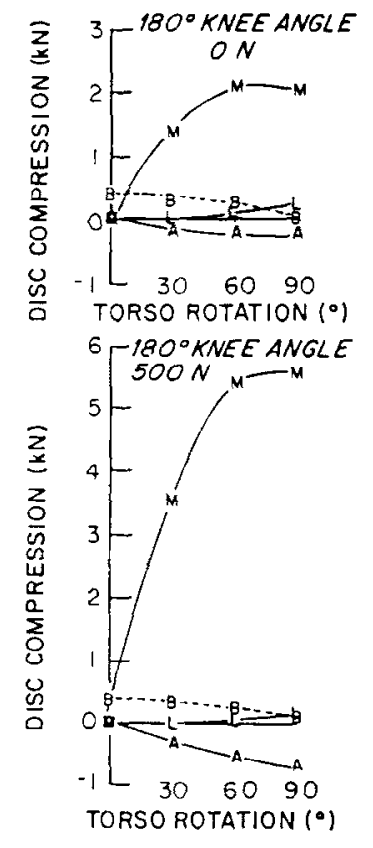

Fig. 11. Disc compression vs torso rotation by element at $0 \mathrm{~N}$ and $500 \mathrm{~N}$ load in the hands with $180^{\circ}$ knee angle (straight leg).
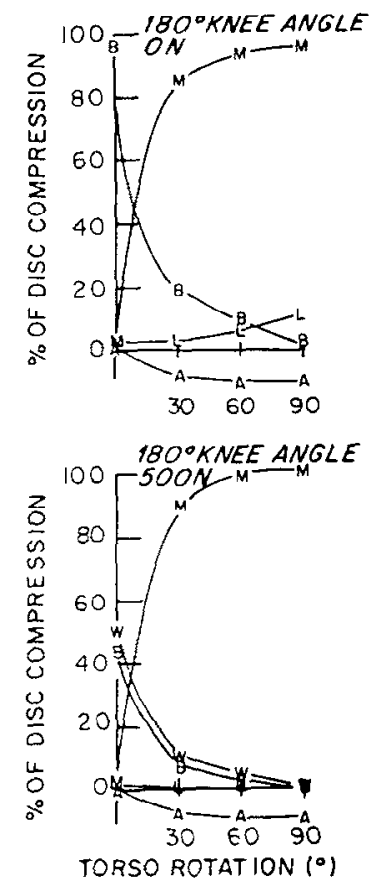

Fig. 12. Percent of disc compression vs torso rotation by element at $0 \mathrm{~N}$ and $500 \mathrm{~N}$ load in the hands with $180^{\circ} \mathrm{knee}$ angle (straight leg).

a negligible impact on compressive force, so only one condition $\left(180^{\circ}\right)$ is displayed.

The first observation to be made is that compression increases as the trunk is flexed. It can also be seen that muscle contraction represents the major contribution to disc compression at $30^{\circ}$ or more of torso rotation while at less than $30^{\circ}$ the effect of body weight and load in the hands predominates. At $0 \mathrm{~N}$ load the ligaments generate a relatively significant percent of the total compression, but their effect is virtually negated at the $500 \mathrm{~N}$ load (see Fig. 12) by the muscle forces. Abdominal pressure, which acts as a parallel compression-bearing element to the disc, never relieves more than about $10 \%$ of the compressive force. This is far below the $40^{\circ}{ }_{10}$ estimated by Eie (1966) but fairly close to the estimate of $15-20^{\circ}$ o put forth by Chaffin (1982) and $14^{\circ}$ proposed by Schultz et al. (1982).

The magnitude of the load in the hands also strongly affects the magnitude of the disc compression. A $500 \mathrm{~N}$ load in the hands leads to at least a doubling of disc compression over no-load conditions. The maximum level increases from approximately $2200 \mathrm{~N}$ to $5400 \mathrm{~N}$. NIOSH has stated that disc compressive loads above $3425 \mathrm{~N}$ should be avoided if possible in order to reduce the risk of low-back pain (NIOSH, 1980). This recommendation was based in part on the observations of Evans and Lissner (1965), Sonoda (1962) and Perey (1957) who found vertebral endplate microfractures at loads starting at roughly $2500 \mathrm{~N}$ and above, particularly for older individuals. Therefore the model suggests that excessive back compressive forces can arise under the conditions of this study.

In general, the model predictions all follow the patterns that would be anticipated or seem reasonable for changes in torso angle, load and knee angle. Therefore the results of the analysis of posture and load further substantiate the validity of this integrative biomechanical model.

\section{SUMMARY}

A biomechanical model has been developed that concurrently estimates strains on ligaments in the low. back region, trunk erector moment requirements, L5/S1 annulus strain, and sacral endplate compression for static, sagittal-plane two-handed symmetric lifting tasks.

Validation studies suggest that the model yields reasonable predictions based on comparisons to experimental studies and results of sensitivity analyses. More information is needed, though, in order to better model the articular ligament in that the current data leads to unreasonably high levels of maximum strain. It is also important to pursue the load-bearing role of the facet joints.

Model sensitivity analyses yielded the following observations:

(1) ligament strains are typically well within the transition region (for those ligaments on which stress-strain curves are published);

(2) the strains on the lumbodorsal fascia come the closest to the upper bound of their transition region;

(3) strains in the articular ligaments may be excessive but it is difficult to discern because the predicted 
values suggest that better modeling information is needed;

(4) maximum ligament strains for any given trunk flexion angle occur when bending forward with knees flexed and no load in the hands;

(5) maximal strains on the posterior aspect of the annulus remain below $3 \%$ for typical industrial lifting tasks, which is a value falling well within the toe region of this tissue's stress-strain curve;

(6) industrial materials handling moment requirements for the trunk erector musculature reach levels that exceed the strength capabilities of a significant proportion of the workforce population in terms of isometric strength values and

(7) disc compressive forces could reach levels well above those known to cause failure of the vertebral cartilage endplate from cadaver specimens.

It is concluded, on the basis of model sensitivity analyses, that typical lifting tasks can lead to excessive disc compressive forces, muscle moment requirements and possibly lumbodorsal fascia strains. At the other end of the spectrum, annulus rupture due directly to strain in the lamina appears unlikely, except where the annulus has suffered prior degeneration.

\section{REFERENCES}

Adams, M. A., Hutton, W. C. and Stott, J. R. R. (1980) The resistance to flexion of the lumbar intervertebral joint. Spine 5, 245-253.

Anderson, C. (1983) A biomechanical model of the lumbosacral joint for lifting activities. Ph.D. Thesis, Department of Industrial and Operations Engineering, The University of Michigan.

Anderson, C., Chaffin, D. and Herrin, G. (in press) A study of lumbosacral orientation during load lifting. Spine.

Andersson, G. B. J., Herberts, P. and Ortengren, R. (1976) Myoelectric back muscle activity in standardized lifting postures. Biomechanics 5-A (E.jited by Komi, P. V.). University Park Press, Baltimore.

Andersson, G. B. J., Ortengren, R. and Herberts, P. (1977) Quantitative electromyographic studies of back muscle activity related to posture and loading. Orthop. Clin $N \mathrm{Am}$. 8, 85-96.

Ayoub, M. M., Bethea, N. J., Deivanayagam, S., Asfour, S. S., Bakken, G. M., Liles, D., Mital, A. and Sherif, M. (1978) Determination and modelling of lifting capacity. NIOSH, HEW Final Report for Grant No. 5 ROL OH 00545-02.

Bazergui, A., Lamy, C. and Farfan, H. F. (1978) Mechanical properties of the lumbodorsal fascia. Paper no. IA-08 Proceedings of the 1978 Society for Experimental Stress Analysis Meeting, Wichita, KS.

Broberg. K. B. (1983) On the mechanical behaviour of intervertebral discs. Spine 8, 151-165.

Broberg, K. and von Essen, H. (1980) Model of intervertebral discs. Spine 5, 155-167.

Brown, T., Hansen, R. J., and Yorra, A. J. (1957) Some mechanical tests on the lumbosacral spine with particular reference to the intervertebral disc. J. Bone Jt Surg. 39A, $1135-1164$.

Chaffin, D. (1969) Computerized biomechanical modelsdevelopment and use in studying gross body actions. J. Biomechanics 2, 429-441.

Chaffin, D. (1982) Low-back stresses during load lifting Human Body Dynamics: Impact, Occupational and Athletic
Aspects (Editcd by Ghista, D. V.). Clarendon Press, Oxford

Chaffin, D. B. and Andersson, G. B. J. (1984) Occupational Biomechanics. John Wiley, New York.

Eie. N. (1966) Load capacity of the low back. J. Oslo Cy Hoss 16, 75-98.

Evans, F. G. and Lissner, H. R. (1965) Studies on the energy absorbing capacity of human lumbar intervertebral discs. Proceedings of the Setenth Stapp Car Crash Conference, Springfield, IL.

Farfan, H. F. (1973) Mechanical Disorders of the Low Back. Lea and Febiger, Philidelphia.

Fisher, B. (1967) A biomechanical model for the analysis of dynamic activities. Masters Thesis, Department of Industrial and Operations Engineering, The University of Michigan.

Galante, J. O. (1967) Tensile properties of the human lumbar annulus fibrosis. Acta orthop. scand., suppl. 100.

Garg, A. and Chaffin, D. (1975) A biomechanical computerized simulation of human strength. AIIE Trans. 7, $1-15$.

Glover, J. R. (1960) Back pain and hyperaesthesia. Lancet 1, $1165-1169$.

Gracovetsky, S., Farfan, H. F. and Lamy, C. (1981) The mechanism of the lumbar spine. Spine 6, 249-262.

Hickey, D. S. and Hukins, D. W. (1980) Relation between the structure of the annulus fibrosus and the function and failure of the intervertebral disc. Spine 5, 106-116.

Katake, K. (1961) The strength for tension and bursting of human fasciae. J. Kyoto Pref. Med. Unit. 69, 484-488.

Morris, J. M., Lucas, D. B. and Bresler, B. (1961) Role of the trunk in stability of the spine. J. Bone Jt Surg. 43A, 327-351.

Nachemson, A. (1963) The influence of spinal movements on the lumbar intradiscal pressure and on the tensile stresses in the annulus fibrosus. Acta orthop. scand. 33, 183-207.

Nachemson, A. and Evans, J. (1968) Some mechanical properties of the third lumbar inter-laminar ligament (ligamentum flavum). J. Biomerhanics 1, 211-220.

NIOSH (1980) A Work Practices Guide For Manual Lifting. National Institute for Occupational Safety and Health, Taft Laboratories, Cincinnati.

Panjabi, M. M., Krag, M. H., White, A. A. and Southwick, W. O. (1977) Effects of preload on load displacement curves of the lumbar spine. Orthop. Clin. N. Am. 8, 181-192.

Perey, O. (1957) Fracture of the vertebral endplate in the lumbar spine, an experimental biomechanical investigation. Acta orthop. scand. suppl. 25.

Rissanen, P. (1960) The surgical anatomy and pathology of the supraspinous and interspinous ligaments of the lumbar spine with special reference to ligament ruptures. Acta orthop. scand. suppl. 46.

Rolander, S. D. (1966) Motion of the lumbar spine with special reference to the stabilizing effect of posture fusion. Acta orthop. scand. suppl. 99.

Schultz, A., Andersson, G., Ortengren, R., Haderspeck, K. and Nachemson, A. (1982) Loads on the lumbar spine: validation of a biomechanical analysis by measurements of intradiscal pressures and myoelectric signals. $J$. Bone $J t$ Surg. 64A, 713-720.

Schultz, A., Belytschko, T. B., Andriacchi, T. P. and Galante, J. O. (1973) Analog studies of forces in the human spine: mechanical properties and motion segment behavior. $J$. Biomechanics 6, 373-383.

Schultz, A. B., Warwick, D. N., Berkson, M. H. and Nachemson, A. L. (1979) Mechanical properties of human lumbar spine motion segments-Part 1: Responses in flexion, extension, lateral bending and torsion. J. biomech. Engng. 101, 46-52.

Seager, F. G. N. (1959) Some observations on the incidence and treatment of back injuries in industry. Ann. occup. $H y g$. $1,180-185$

Seireg, A and Arvikar, R. J. (1975) A comprehensive musculo- 
skeletal model for the human vertebral column. Adiances In Bioengineering (Edited by American Sociely of Mechanical Engineers). ASME, New York.

Silver, P. H. S. (1954) Direct observation of changes in tension in the supraspinous and interspinous ligaments during flexion and extension of the vertebral column in man. $J$. Anat. 88, 550-551

Sonoda. T. (1962) Studies on the compression, tension and torsion strength of the human vertebral column. J. Kyoto Pref. Med. Unit. 71, 659-702.

Spilker, R. L. (1980) Mechanical behavior of a simple model of an intervertebral disc under compressive loading. J. Biomechanics 13,895-901.

Takashima, S. T, Singh. S. P., Haderspeck, K. A. and Schultz, A. B. (1979) A model for semi-quantitative studies of muscle actions. J. Biomechanics 12, 927-939.

Troup, J. D. G. (1965) Relation of lumbar spine disorders to heavy manual work and lifting. Lancet 1, 857-861.

Waters, R. L. and Morris, J. M. (1973) An in vitro study of normal and scoliotic interspinous ligaments. J. Biomechanics 6, 343-348.

Yettram. A. L. and Jackman. M. J. (1980) Equilibrium analysis for the forces in the human spinal column and its musculature. Spine 5, 402-4l1.

\section{APPENDIX A. ELEMENT RESTING LENGTHS}

Resting lengths for the ligaments were determined by calculating the distance between the attachment points on the two vertebra and then adjusting for any pre-tension. Adjustments were also made for any disc deflection resulting from compression due to ligament strain. Resting length unless otherwise noted was assumed to occur in the erect posture with no load in the hands (Adams et al., 1980). A pre-strain of $15^{\prime \prime}$ "was incorporated for the ligamentum flavum (Nachemson and Evans, 1968) and it was assumed that the supraspinous/interspinous ligament dues not come into tension until halfway to full trunk flexion (Adams et al. 1980; Nachemson and Evans, 1968; Silver, 1954). Farfan (1973) notes that there is typically $12^{\circ}$ of rotation between $\mathrm{L} 5$ and $\mathrm{S} I$ at full-flexion, so it was assumed that the resting length of the supraspinous/interspinous ligament occurred at $6^{\prime}$ flexion between $L 5$ and $\$ 1$. Resting lengths by ligament and sex are given in Table 3
Table 3. Ligament resting lengths by sex

\begin{tabular}{llc}
\hline \multicolumn{1}{c}{ Ligament } & \multicolumn{2}{c}{ Resting length (cm) } \\
& Male & Female \\
\hline Interspinous/supraspinous & 5.037 & 4.496 \\
Iliolumbar, sacrolumbar & 3.856 & 3.409 \\
Articular & 0.657 & 0.529 \\
Ligamentum flavum & 4.863 & 4.393 \\
Lumbodorsal fascia & 4.434 & 3.944 \\
\hline
\end{tabular}

\section{APPENDIX B: LIGAMENT FORCE-STRAIN EQUATIONS}

Experimentally-derived force-deformation and stress strain data are available for the ligamentum flavum, interspinous superspinous ligaments, and the lumbodorsal fascia. The data sources are as shown in Table 2 . These data were mathematically modelled using an exponential equation of the form

$$
F=a e^{h s}
$$

where $F=$ force in ligament, $s=$ percent strain in ligament and $a, b$ are coefficients.

Curves were fit to the available data using a linear regression model on the logarithm transform of equation (B1). For the lumbodorsal fascia the curve for thigh fascia from Katake (1961) was used to obtain the equation form, but the values were scaled to the elastic limit data provided by Bazergui $e l$ al. (1978). The number of points for each ligament ranged from 5 to 9 so it is not surprising that coefficients of determination were between 0.98 and 1 . The equations are as follows:

interspinous/supraspinous ligament

$$
F=2.3296 e^{0:-215 s}
$$

ligamentum flavum

$$
F=1.1994 e^{000432 S}
$$

lumbodorsal fascia

$$
F=0.53819 e^{0.17843 S}
$$

where $F=$ force in ligament $(\mathrm{N}), S=$ strain in ligament $\left({ }^{\circ}{ }_{0}\right)$. Experimental force deformation or stress-strain data is

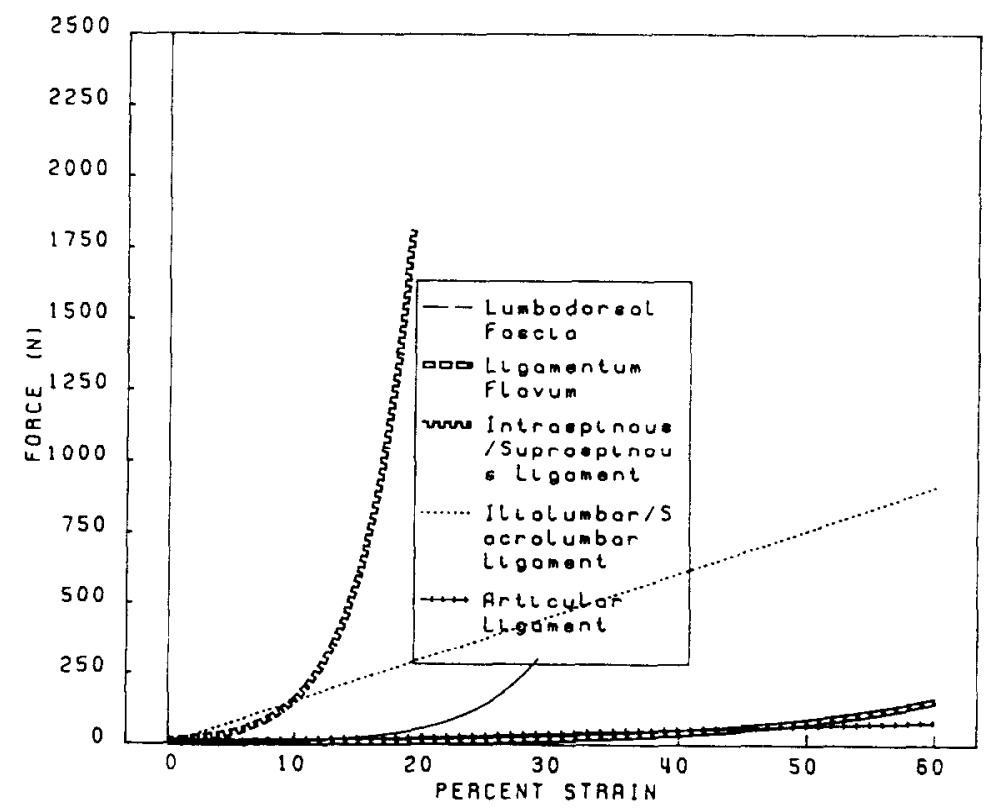

Fig. 13. Predicted force vs strain for ligaments in the lumbosacral biomechanical model. 
not available on the articular or iliolumbar sacrolumbar ligaments so the estimates of Schultz et al. (1973) and Takashima et al. (1979) were adopted. The equations are as follows:

articular ligaments (both)

$$
F=1.3377 \mathrm{~S}
$$

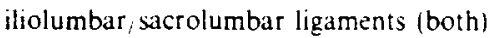

$$
F=15.221 \mathrm{~S} \text {. }
$$

Predicted force vs strain curves are shown in Fig. 13. It can be seen that there is a wide range of material properties for the various ligaments.

\section{APPENDIX C: DISC MODEL PARAMETERS}

The disc model of Broberg and von Essen (1980) models the disc as a cylinder-like object with a concentric nucleus. There are eleven laminae in the annulus. In each lamina the annulus fibers wrap around the walls of the cylinder at a specified inclination to the base. In the resting state the outermost layer bulges a specified amount. Therefore the physical structure of the disc is described by its cross-sectional area, height, nucleus-to-disc cross-sectional area ratio. fiber angle and initial bulge. The values for these parameters were selected on the basis of literature review and sensitivity analyses and are summarized in Table 4
Table 4. Summary of disc parameters by sex

\begin{tabular}{lcc}
\hline \multicolumn{1}{c}{ Parameter } & Male disc & Female disc \\
\hline $\begin{array}{l}\text { Cross-sectional area } \\
\text { Disc height }\end{array}$ & $17.73 \mathrm{~cm}^{2}$ & $16.77 \mathrm{~cm}^{2}$ \\
$\begin{array}{l}\text { Cross-sectional } \\
\text { area ratio }\end{array}$ & $12.73 \mathrm{~mm}$ & $11.19 \mathrm{~mm}$ \\
$\begin{array}{l}\text { Fiber angle } \\
\text { Initial bulge of } \\
\text { outermost layer }\end{array}$ & $50 \%$ & $50 \%$ \\
\hline
\end{tabular}

The model allows consideration of discs with linear or nonlinear relations between fiber strain and stress and homogeneous or inhomogeneous spacing between the lamina. Sensitivity analyses performed on the model using the four options formed by combinations of linear/nonlinear and homogeneous/inhomogeneous discs suggested that a nonlinear homogeneously spaced annulus yielded predicted behavior most like experimental results. The shape of the nonlinear relation between stress and strain is the following

$$
G(S)=S+100 S^{2}
$$

where $G(S)=$ stress as a function of strain and $S=$ strain on annulus fiber.

The curve is scaled by a constant so that the model behavior best mimics experimental resulis. Details on the sensitivity analyses can be found in Anderson (1983). 